\title{
Dakota Uncertainty Quantification Methods Applied to the CFD code Nek5000
}

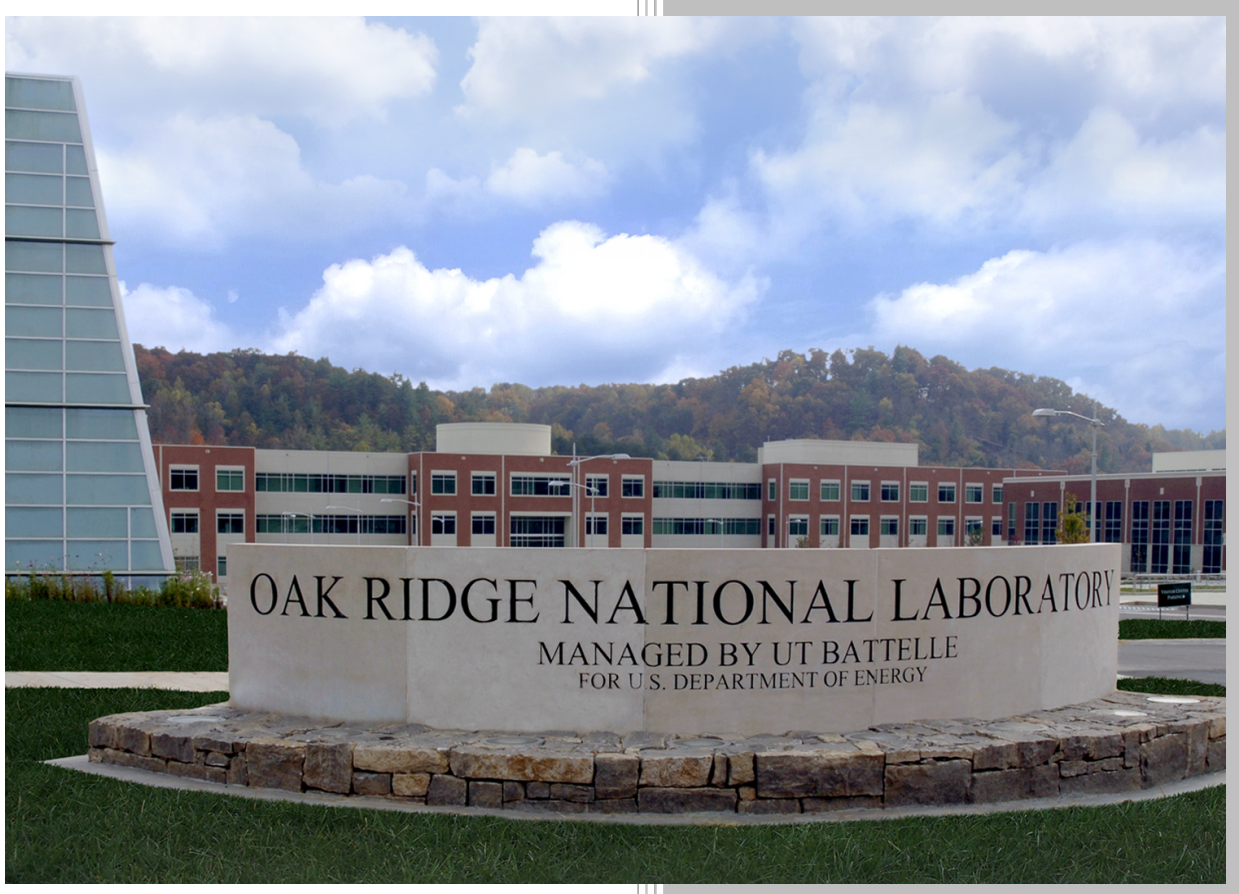

Marc-Olivier Delchini Emilian L. Popov

William David Pointer

04/29/2016 


\title{
DOCUMENT AVAILABILITY
}

Reports produced after January 1, 1996, are generally available free via US Department of Energy (DOE) SciTech Connect.

Website: http://www.osti.gov/scitech/

Reports produced before January 1, 1996, may be purchased by members of the public from the following source:

\author{
National Technical Information Service \\ 5285 Port Royal Road \\ Springfield, VA 22161 \\ Telephone: 703-605-6000 (1-800-553-6847) \\ TDD: $703-487-4639$ \\ Fax: 703-605-6900 \\ E-mail: info@ntis.fedworld.gov \\ Website: http://www.ntis.gov/help/ordermethods.aspx
}

Reports are available to DOE employees, DOE contractors, Energy Technology Data Exchange representatives, and International Nuclear Information System representatives from the following source:

Office of Scientific and Technical Information

PO Box 62

Oak Ridge, TN 37831

Telephone: $865-576-8401$

Fax: 865-576-5728

E-mail: report@osti.gov

Website: http://www.osti.gov/contact.html

This report was prepared as an account of work sponsored by an agency of the United States Government. Neither the United States Government nor any agency thereof, nor any of their employees, makes any warranty, express or implied, or assumes any legal liability or responsibility for the accuracy, completeness, or usefulness of any information, apparatus, product, or process disclosed, or represents that its use would not infringe privately owned rights. Reference herein to any specific commercial product, process, or service by trade name, trademark, manufacturer, or otherwise, does not necessarily constitute or imply its endorsement, recommendation, or favoring by the United States Government or any agency thereof. The views and opinions of authors expressed herein do not necessarily state or reflect those of the United States Government or any agency thereof. 
Reactor and Nuclear Systems Division

\title{
Dakota Uncertainty Quantification Methods Applied to the CFD code Nek5000
}

\author{
Marc-Olivier Delchini, Emilian L. Popov, William David Pointer
}

Date Published: 06/2016

\author{
Prepared by \\ OAK RIDGE NATIONAL LABORATORY \\ P.O. Box 2008 \\ Oak Ridge, Tennessee 37831-6285 \\ managed by \\ UT-Battelle, LLC \\ for the \\ US DEPARTMENT OF ENERGY \\ under contract DE-AC05-00OR22725
}





\section{CONTENTS}

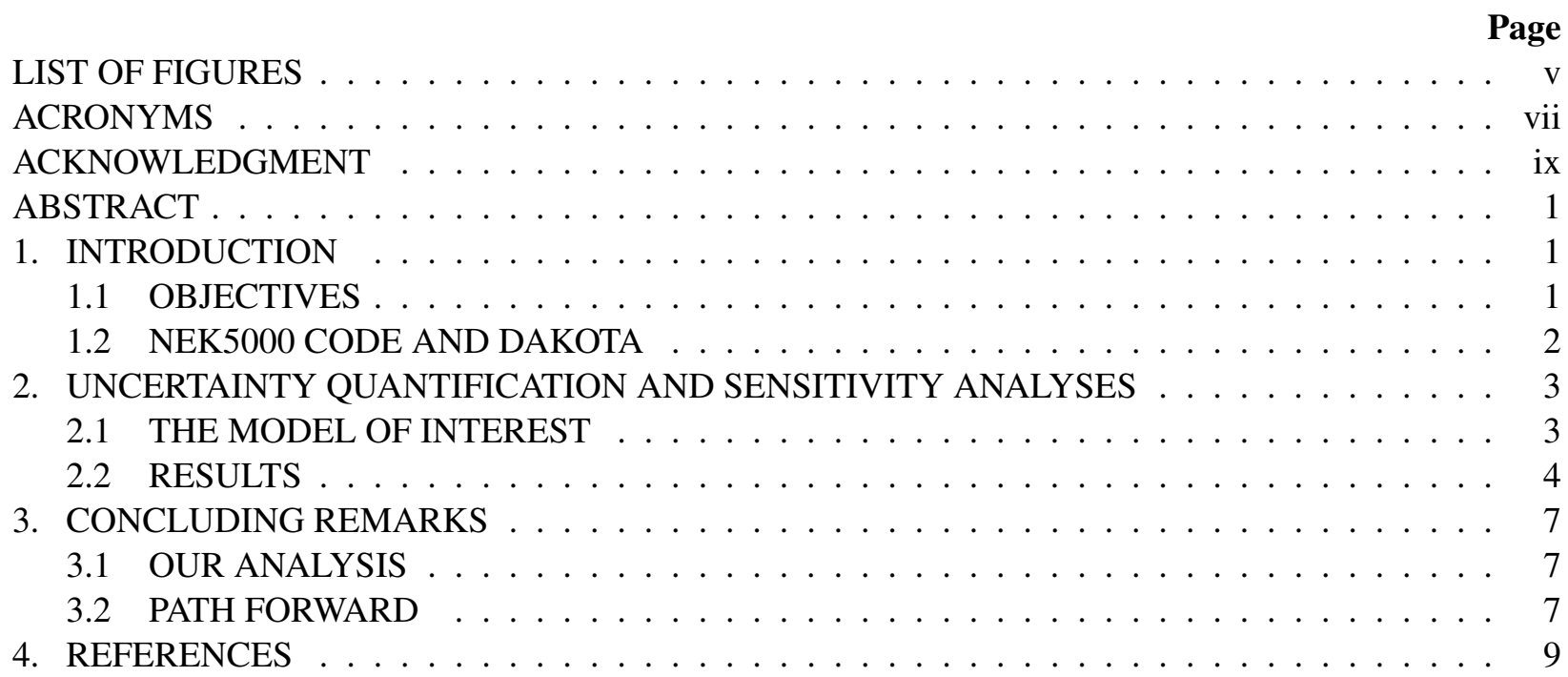





\section{LIST OF FIGURES}

Figures $\quad$ Page

2 Velocity magnitude profile in a slice located at $(\boldsymbol{x}, \boldsymbol{y}, \boldsymbol{z})=(\mathbf{0 , 0}, \mathbf{7 0})$ for different values of

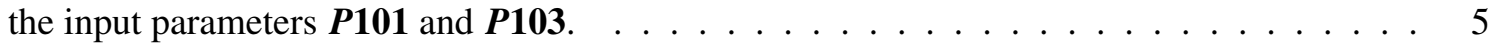

3 Time average velocity magnitude profile on slice $\mathrm{S} \ldots \ldots \ldots \ldots$ 



\section{ACRONYMS}

NEAMS Nuclear Energy Advanced Modeling and Simulation

CFD computational fluid dynamics

UQ uncertainty quantification

LES large eddy simulation

URANS unsteady Reynolds average Navier-Stokes

SEM spectral element method 



\section{ACKNOWLEDGEMENT}

This work was supported by the Nuclear Energy Advanced Modeling and Simulation (NEAMS) program under the auspices of the US Department of Energy, Office of Nuclear Energy. 



\begin{abstract}
This report presents the state of advancement of a Nuclear Energy Advanced Modeling and Simulation (NEAMS) project to characterize the uncertainty of the computational fluid dynamics (CFD) code Nek5000 using the Dakota package [2] for flows encountered in the nuclear engineering industry. Nek5000 is a high order spectral element CFD code developed at Argonne National Laboratory for high resolution spectral-filtered large eddy simulations (LESs) and unsteady Reynolds averaged Navier-Stokes (URANS) simulations. The Dakota package developed at Sandia National Laboratory can be integrated with many scientific and engineering codes to facilitate efficient, effective uncertainty quantification (UQ) and sensitivity analyses. The objective of this work is to perform a UQ and a sensitivity analysis of the numerical and physical models implemented in Nek5000 and used to solve for flows in two geometries of interest: a 3-D pipe and a 7-pin bundle. The results presented in this report demonstrate loose integration of Dakota with Nek5000 and thus should not be used to assess the accuracy of the numerical methods and physical models implemented in Nek5000. All results were obtained by running the default version of Nek5000 which does not include any turbulent models. Thus, any experimental data with the current results is not expected to be validated.
\end{abstract}

\title{
1. INTRODUCTION
}

Predictive modeling and simulation of the performance of a nuclear reactor and its fuel is a challenging task because of the large number of coupled physical phenomena that must be addressed. In addition to this intrinsic complexity, model uncertainty must be accounted for in any analysis if the model will be used to facilitate design or operational decisions which may impact safety and performance. Rigorous, structured uncertainty analyses are performed by first characterizing the model's input uncertainties and, then propagating the uncertainty through the model in order to estimate the output's uncertainty.

\subsection{OBJECTIVES}

This project is part of the ongoing effort to assess modeling uncertainty in Nek5000 simulations of flow configurations relevant to the advanced reactor applications of the NEAMS program. Two geometries are under investigation in these preliminary assessments: a 3-D pipe and a 3-D 7-pin-bundle. Initial efforts have focused on gaining an understanding of Nek5000 modeling options and integration of Nek5000 with Dakota. This report focuses on an initial demonstration of the use of Dakota to assess parametric uncertainties in a simple pipe flow problem. This problem is used to optimize performance of the uncertainty quantification strategy and to estimate computational requirements for assessments considering more complex geometries.

The objective of this project is threefolds. In the first step, the uncertainty of the numerical model, the flow condition, and the material properties used in Nek5000 will be characterized for flow in a 3-D pipe by comparing the numerical results against experimental data $[4,5]$. Once Nek5000 is calibrated using results from the first study, the flow in a 7-pin-bundle geometry will be simulated. Then, both an uncertainty quantification and a sensitivity analysis will be performed. Particular attention will be focused on UQ methods used (polynomial chaos or sampling methods for instance) in order to minimize the computational cost. In this report, only results of the first step are presented. Comparison with the commercial CFD code Star-CCM+ will be also investigated. 


\subsection{NEK5000 CODE AND DAKOTA}

The computational fluid dynamic (CFD) code Nek5000 developed at Argonne National Laboratory is used to simulate flow behavior in the 3-D pipe. The incompressible Navier-Stokes equations are solved on a computational domain discretized by a spectral element method (SEM). Several temporal discretization methods are available for transient simulations. A second-order temporal discretization is used for the results presented in this report. The Nek5000 code is designed to run on massively supercomputers and has been widely used and tested for CFD simulations around the world.

The UQ analysis is performed using the Dakota package [2] that includes methods for parameter studies, optimization, sensitivity analysis, and uncertainty quantification (UQ). Dakota is designed to interface with external packages/codes such as Nek5000 through a script-driven interface called fork. Such interface provides maximum flexibility to the users for pre- and post-processing tasks preceding and following each function evaluation. For this study, a given set of input parameters of known uncertainty is supplied to Nek5000 through the Dakota interface, and then the code which corresponds to a function evaluation is run. Outputs of interest, or response functions in UQ terms, are returned to Dakota to determine the outputs' uncertainty. More details regarding the input and output parameters of interest are provided in Section 2. 


\section{UNCERTAINTY QUANTIFICATION AND SENSITIVITY ANALYSES}

In this section, details regarding the geometry, as well as the input and output parameters, are presented.

\subsection{THE MODEL OF INTEREST}

Flow of Reynolds number $R e=31 \cdot 10^{3}$ in a 3-D pipe with a nondimensional length of 70 in the z-direction is investigated using the code Nek5000; the time-dependent incompressible Navier-Stokes equations are solved on an unstructured mesh of 200, 000 hexagonal elements using fourth-order SEM (see Figs. 1a and 1b). Note that the mesh density is high enough to resolve the eddies.

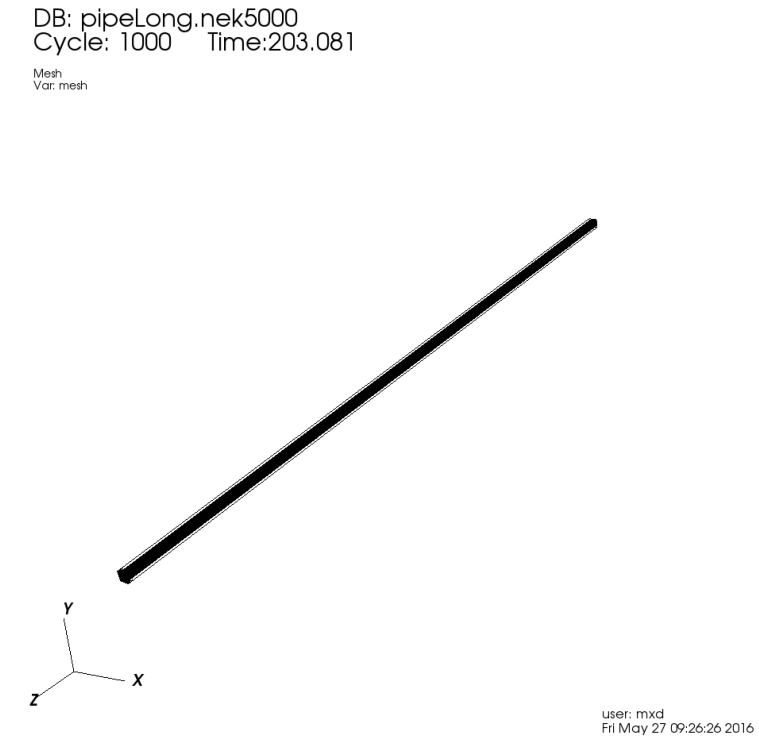

(a) View of the 3-D pipe.

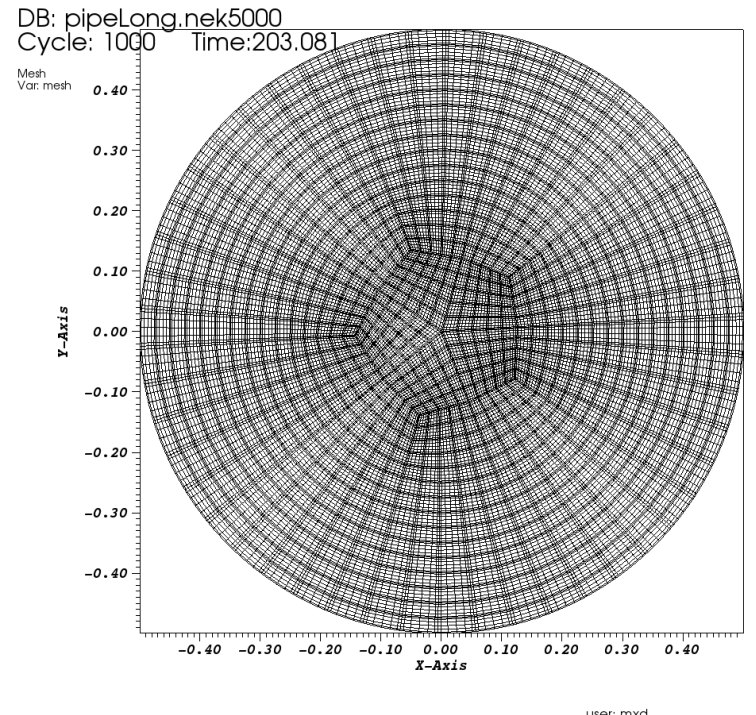

(b) Mesh density in a slice of the 3-D pipe.

For each time step, an estimate of the velocity profile is obtained through a Helmholtz solver, and then corrected by solving pressure Laplace equations. Inlet and outflow boundary conditions are used to set the inlet velocity profile and the outlet pressure, respectively.

We ran the large eddy simulation (LES) version of Nek5000 and monitor the $L_{2}$ error norm between the numerical and experimental data describing the fully developed radial velocity profile. The $L_{2}$ norm is computed using the following four-step process (all length are in nondimensional unit):

1. The time-averaged, $\mathrm{z}$-direction velocity is extracted from the Nek5000 output file from a slice at $(\mathrm{x}, \mathrm{y}, \mathrm{z})=(0,0,70)$ using Visit [1]. This information is collected from 0 to $5 \mathrm{~s}$ and stored in a text file along with node coordinates.

2. For each node of the slice $S$, the distance to the outside wall is computed using the following relation:

$$
\text { dist }_{\text {to wall }}=\frac{D}{2}-\sqrt{\left(x^{2}+y^{2}\right),}
$$

where $D=1$ is the diameter of the pipe in nondimensional unit. 
3. Once the variable dist to wall is obtained, the nodes are gathered in pools using the following rule: a node belongs to a pool if the relative difference between the value $d_{i s t} t_{\text {to }}$ wall (node) and the pool radius $r_{\text {pool }}$ is less than a user-supplied tolerance: $t o l$. When a node meets the previous criterion, the corresponding velocity magnitude $V\left(\right.$ node) is added to the pool velocity $V_{\text {pool }}$. A script representing the above would be:

$$
\begin{aligned}
& \text { for loop over nodes of slice } S \\
& \text { ratio }=\frac{\mid r_{\text {pool }}-d i s t_{\text {to wall }}(\text { node }) \mid}{r_{\text {pool }}} \\
& \text { if ratio } \geq \text { tol } \\
& \quad \text { node does not belong to pool } r_{\text {pool }} \\
& \text { else } \\
& \quad \text { node belongs to pool } r_{\text {pool }} \\
& V_{\text {pool }}+=V(\text { node }) \\
& N_{\text {nodes }}+=1 \\
& \text { endif } \\
& \text { endfor } \\
& V_{\text {pool }} *=1 . / N_{\text {nodes }}
\end{aligned}
$$

Note that the number of nodes, $N_{\text {nodes }}$ added to each pool must be tracked in order to compute the pool's average velocity. The pool radius $r_{p o o l}$ can be set equal to the coordinates of the experimental data. It is, however, preferred to have a sufficient number of pools such as all nodes from numerical data fall inside a pool. The number of nodes stored in each pool depends on the value of the tolerance tol.

4. The experimental and numerical values of the velocity magnitude are now available at the same coordinates and are used to compute the $L_{2}$ norm of the error, as follows:

$$
L_{2}^{\text {error }}=\sqrt{\sum_{i}^{N_{\text {pool }}}\left\|V_{\text {pool }}(i)-V_{\text {exp }}(i)\right\|^{2}},
$$

where $N_{\text {pool }}$ is the number of pool.

5. The $L_{2}^{\text {error }}$ norm value is returned to Dakota.

The method described above to compute the $L_{2}^{\text {error }}$ error norm between the experimental data and the numerical results is not used in this report but will be the focus of future investigations. The process described above is driven by a Python script (APPENDIX A.) and thus does not require any user intervention besides launching the Dakota input file. Once Dakota has collected all $L_{2}^{\text {error }}$ norms from the Nek5000 runs, a uncertainty quantification or statistics assessment is performed.

\subsection{RESULTS}

The results presented in this report should not be used to assess the accuracy of the physical models and the numerical methods implemented in Nek5000, but to demonstrate the interest of integrating Dakota with Nek5000 when simulating engineering flows. 
This report presents an initial assessment of the sensitivity of the Nek5000 numerical results for a flow in a 3-D pipe to the spectral filtering input parameters P101 and P103. When turned on, these two parameters control the filtering of modes for LES-type simulations [3]. The parameters $P 101$ and $P 103$ are varied in the ranges $[0,6]$ and $[0.05,0.2]$, respectively, using the discrete_uncertain_set method from the Dakota package. The parameters were treated as discrete uncertain variables (real or integer) whose values come from a set of admissible elements. The contributing simulation was allowed to complete 5,000 time steps on 96 processors in about 5 hours. The time average velocity profile obtained with Nek5000 is plotted against the experimental data in Fig. 2 for different values of the input parameter $P 101$. A solution obtained with default input parameters $(P 101=0$ and $P 103=0)$ in Nek5000 is used as a reference solution to highlight the sensitivity of the numerical simulations when varying the input parameters $P 101$ and $P 103$.

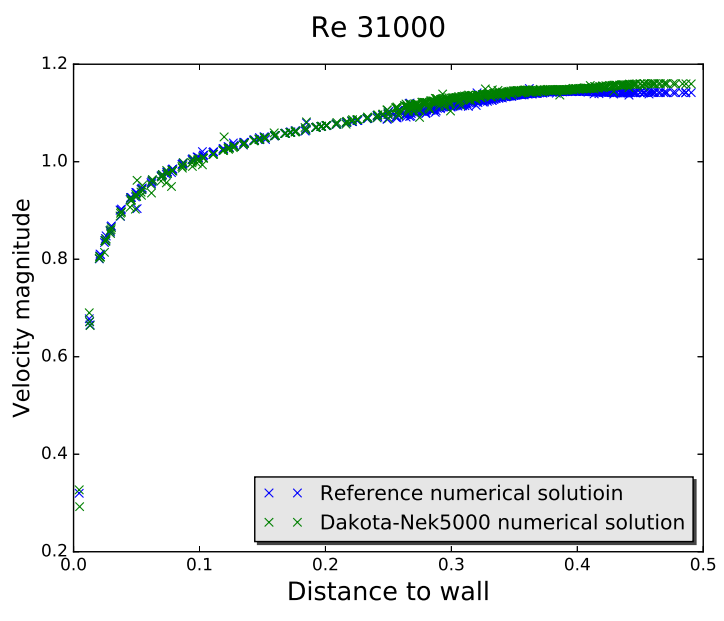

(a) $P 101=0$ and $P 103=5 \cdot 10^{-3}$.

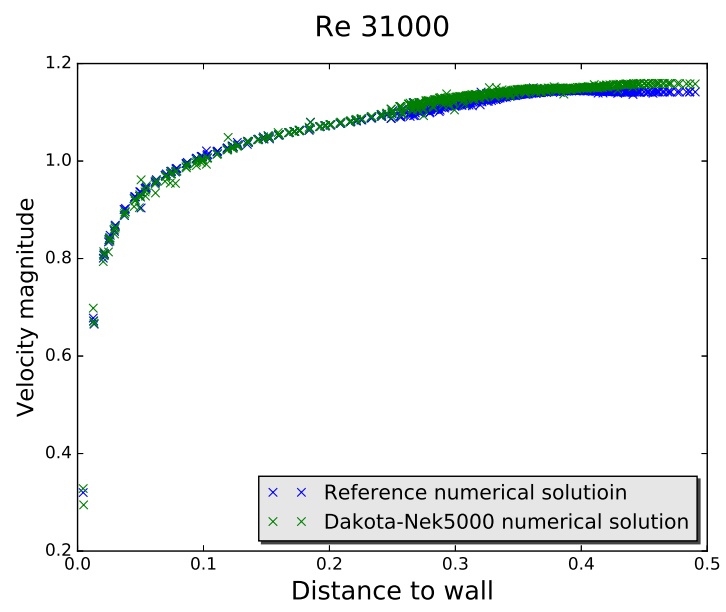

(c) $P 101=4$ and $P 103=5 \cdot 10^{-3}$.

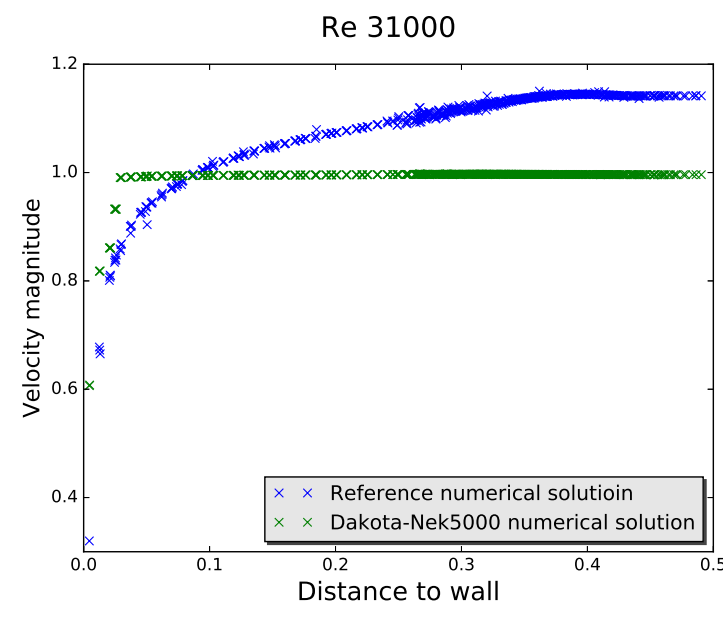

(b) $P 101=2$ and $P 103=5 \cdot 10^{-3}$. Re 31000

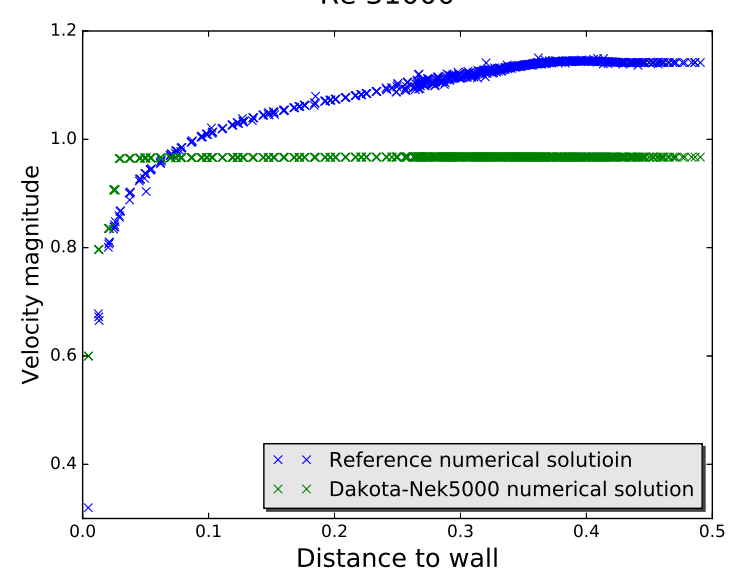

(d) $P 101=6$ and $P 103=5 \cdot 10^{-3}$.

Fig. 2. Velocity magnitude profile in a slice located at $(x, y, z)=(0,0,70)$ for different values of the input parameters $P 101$ and $P 103$.

The numerical results presented in Fig. 2 show a strong dependence upon the parameter $P 101$, i.e. the number of modes filtered when solving for the turbulent flow in the 3-D pipe. As the number of modes 
filtered is increased, the time average velocity profile obtained from Nek5000 diverges from the reference profile. Although it is not shown here, the same behavior is observed when the input parameter $P 103$ increases.

The average velocity magnitude profile on section $S$ is shown in Fig. 3. Turbulence flow is well developed and is consistent with the 1-D velocity profiles presented in Fig. 2: the velocity is zero at the wall, and it increases towards the center of the pipe.

\section{DB: avgpipeLong.nek5000 Cycle: 1000 Time:4.62645}

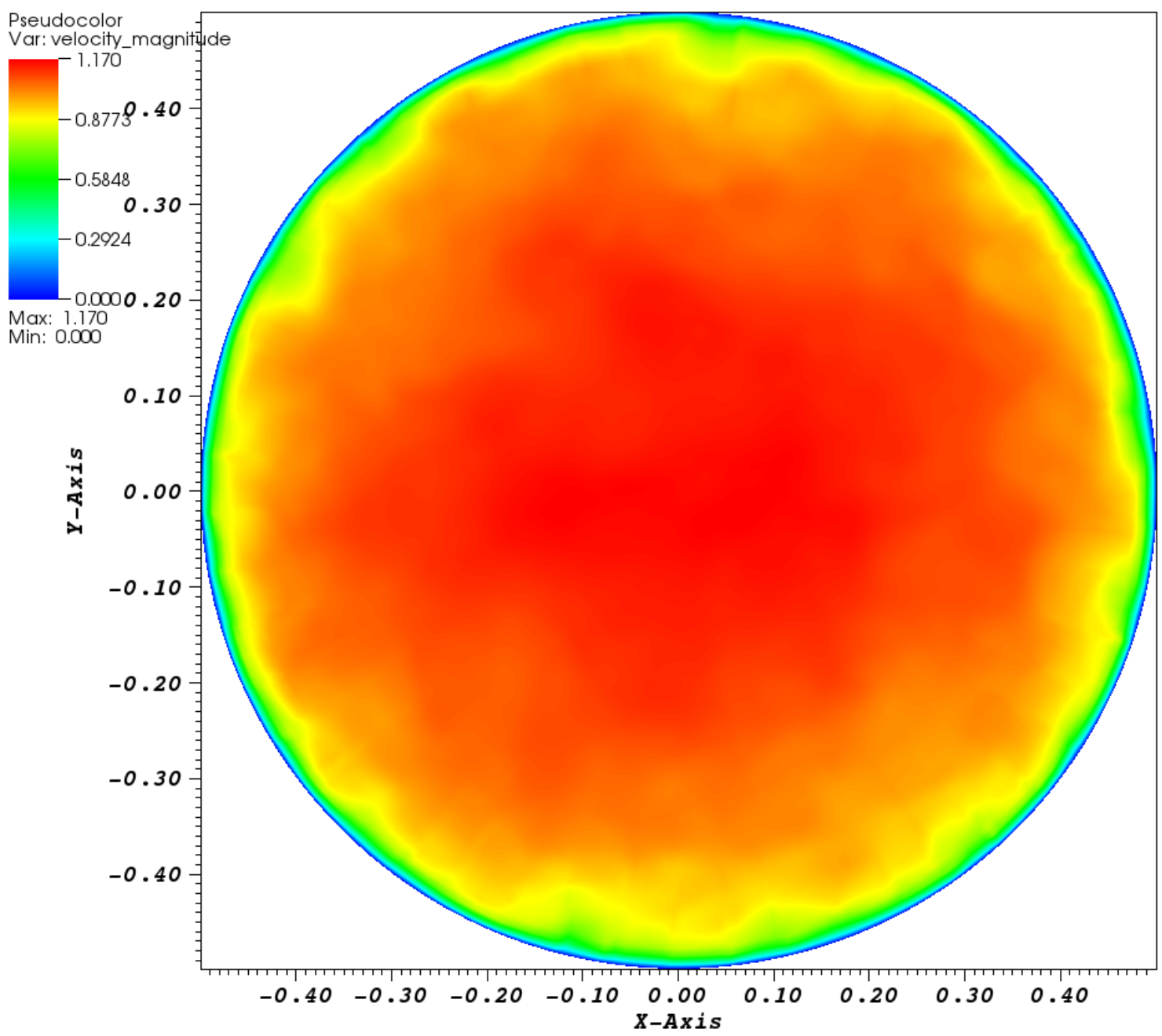

user: $\mathrm{mxc}$

Wed May 4 15:43:53 2016

Fig. 3. Time average velocity magnitude profile on slice $S$. 


\section{CONCLUDING REMARKS}

\subsection{OUR ANALYSIS}

This report demonstrates the sensitivity of Nek5000 predictions of axial velocity in a 3D pipe to input parameters P101 and P103 and outlines an approach for performing a UQ study using Nek5000 and Dakota that will be used in future work. It is estimated that an average of 100 hours computing time on 200 processors will be needed for further UQ analysis.

\subsection{PATH FORWARD}

At this stage, the combined use of Dakota and Nek5000 shows promising results. In the future, the influence of other input parameters on the numerical solution, the computing time, and the number of solver iterations, for instance, will be investigated. A calibration study will be also performed for the flow in a 3-D pipe using the experimental data as reference. In the long term, the same study for flow in a 7-pin bundle will be performed. 



\section{REFERENCES}

\section{References}

[1] “Computer Codes," Lawrence Livermore National Laboratory, https://wci.llnl.gov/simulation/computer-codes/visit/, accessed June 7, 2016.

[2] B. M. Adams, L. E. Bauman, W. J. Bohnhoff, K. R. Dalbey, M. S. Ebeida, J. P. Eddy, M. S. Eldred, P. D. Hough, K. T. Hu, J. D. Jakeman, J. A. Stephens, L. P. Swiler, D. M. Vigil, and T. M. Wildey. Dakota, A Multilevel Parallel Object-Oriented Framework for Design Optimization, Parameter Estimation, Uncertainty Quantification, and Sensitivity Analysis: Version 6.0 User's Manual. Technical report, Sandia National Laboratory SAND2014-4633, (2014).

[3] P. Fisher and J. Mullen. Filter-based stabilization of spectral element method. Numerical Analysis, 332(Serie I):265-270, 2001.

[4] M. V. Zagarola and A. J. Smits. Mean-flow scaling of turbulent pipe flow. J. Fluid Mech., 373(1998):33-79.

[5] M. V. Zagarola and A. J. Smits. "Scaling of the mean velocity profile for turbulent pipe flow". Physical review Letter, 78, 2(1997):349-242. 



\section{APPENDIX A. PYTHON SCRIPT}

\# This python script intends to read a data file (format xmd) and postprocess it. The first part of the code is dedicated to reading and storing the data in arrays that can be easily manipulated in a second step. The second step is user dependent.

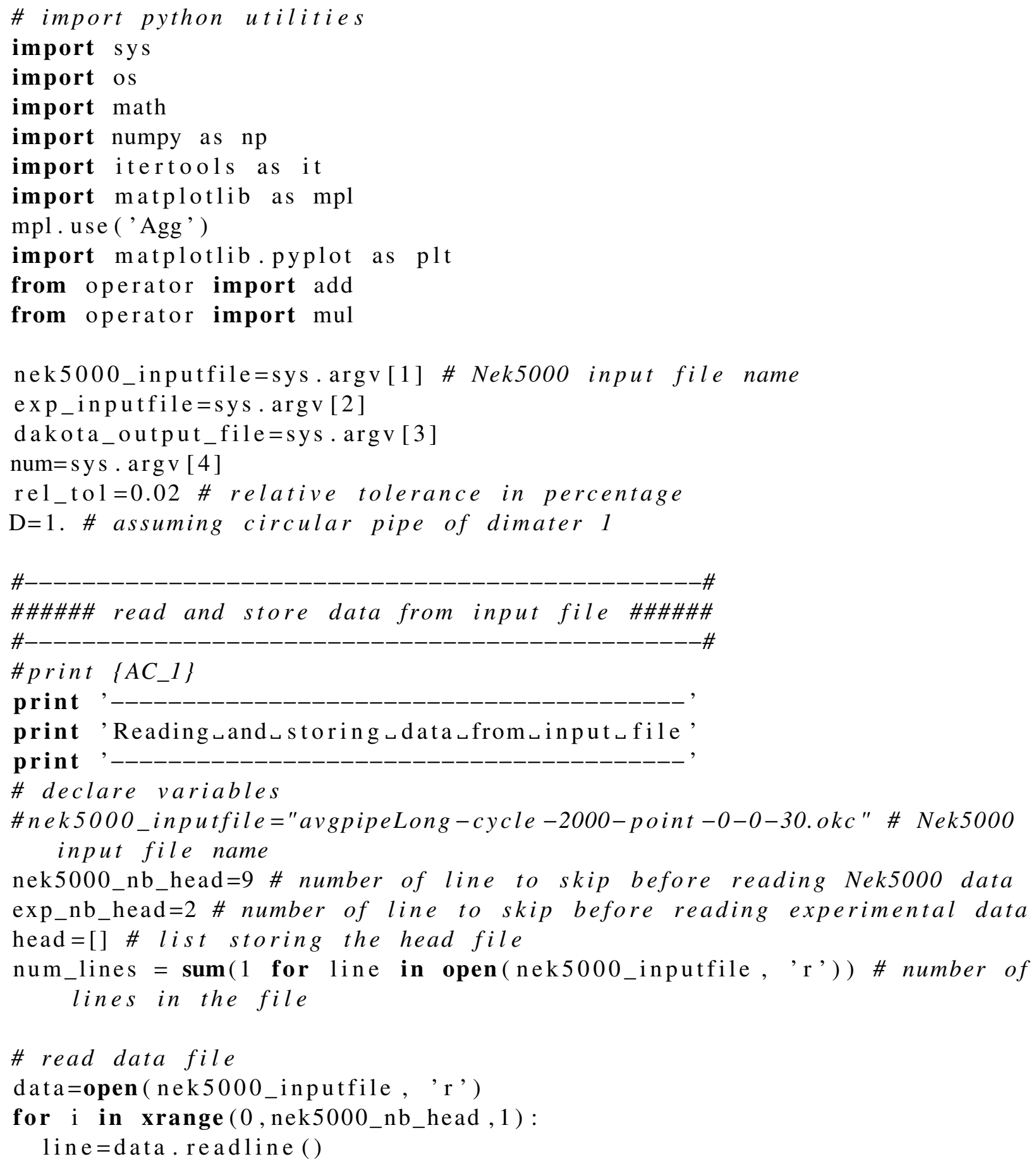


head. append (line.split ())

\# set and print information from head file

nb_var=int (head [0][0]) \# number of variables (including mesh)

print 'Number of $_{\llcorner}$variables:', nb_var

nb_points=int (head [0][1])

nb_phys_var=nb_var-3 \# number of physical variables (excluding mesh)

print 'Number of $_{\llcorner} \mathrm{variables} \leftarrow$ excluding the $_{\llcorner}$mesh:', nb_phys_var

var_names=head [1:nb_var+1] \# variables names from the input file

print 'Variable $\leftarrow$ names:', var_names

max_var=head [nb_var $\left.+1: 2 * \mathrm{nb} \mathrm{n}_{-} \mathrm{var}+1\right]$ \# maximum values for all variables

for $i$ in xrange $\left(0, \mathrm{nb}_{-} \mathrm{var}, 1\right)$ :

print 'Minimum $\_$and maximum $_{\llcorner}$values_for', var_names [i ], ' :', max_var[i ] $[0: 2]$

\# declare variables to store data

$\mathrm{x}=[] \# x$

$\mathrm{y}=[] \# y$

$\mathrm{z}=[] \# z$

$\operatorname{var}=[] \#$ variables

\# loop over the remaining of the file, make them float and store them for i in xrange(nek5000_nb_head,num_lines, 1):

line $=$ data. readline ()

row $=$ line.split ()

$\mathrm{x}$. append (float (row $[0]))$

y. append (float (row [1]))

$\mathrm{Z}$. append (float (row [2]))

var. append (map(float, row [3: nb_var +1]))

print

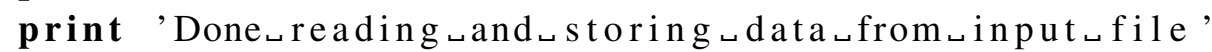

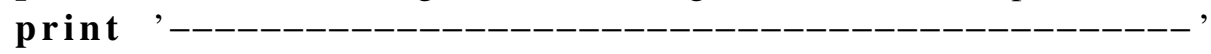

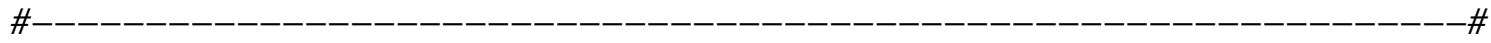

\#\#\#\#\#\#\#\#\#\#\#\#\# post-processing experimental data \#\#\#\#\#\#\#\#\#\#\#\#\#

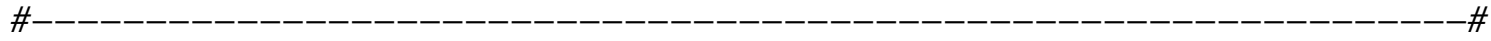

print

print 'Post-processing experimentalıdata',

print '---------------------------------'

\# Read experimental data

exp_dist_to_wall= []

exp_vel $=[]$ 


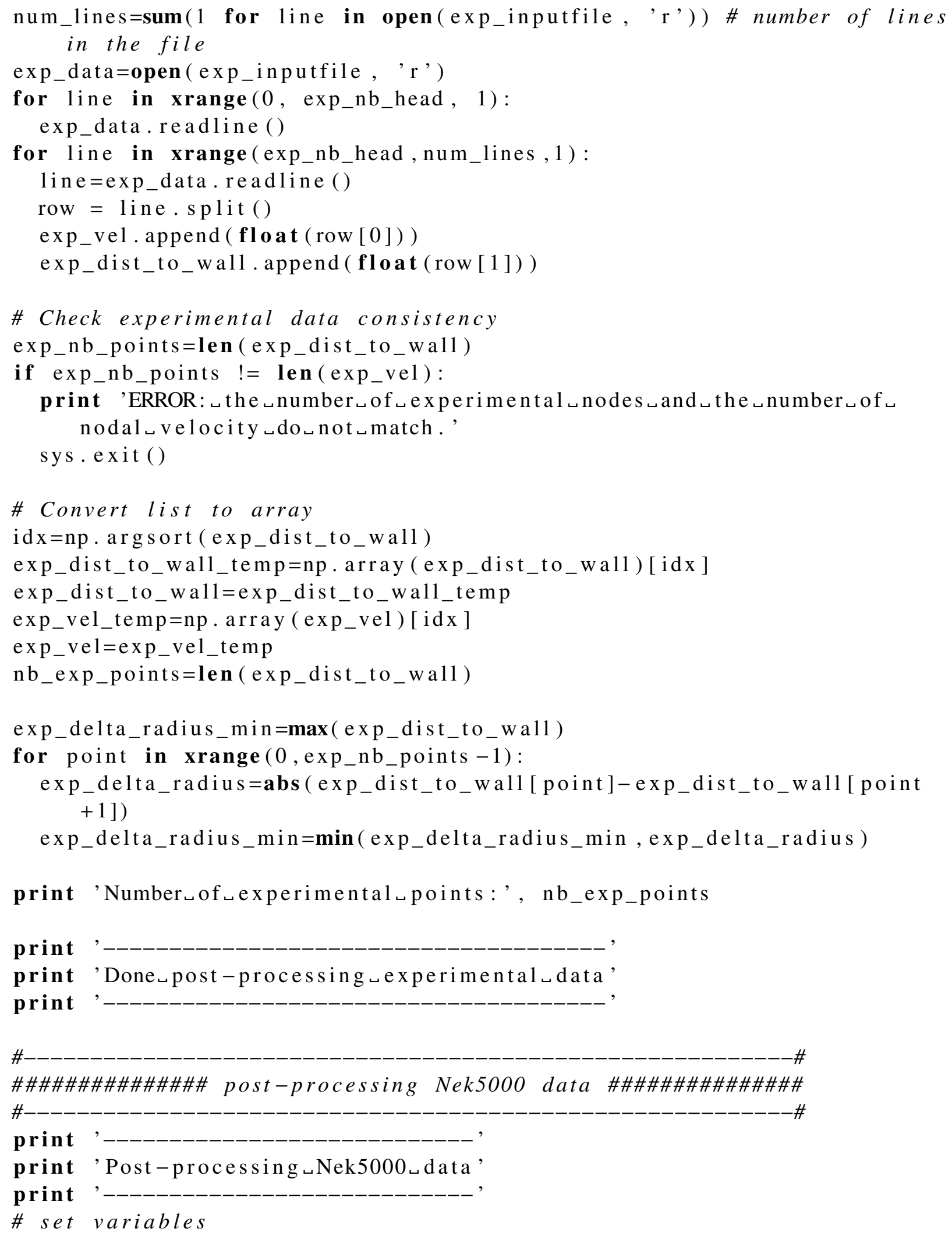


nb_points $=$ len $(x)$

print 'Number of $_{\llcorner}$points/nodes to $_{\llcorner}$post-process:', nb_points

print 'Relative ${ }^{\prime}$ tolerancestosuse:, , rel_tol

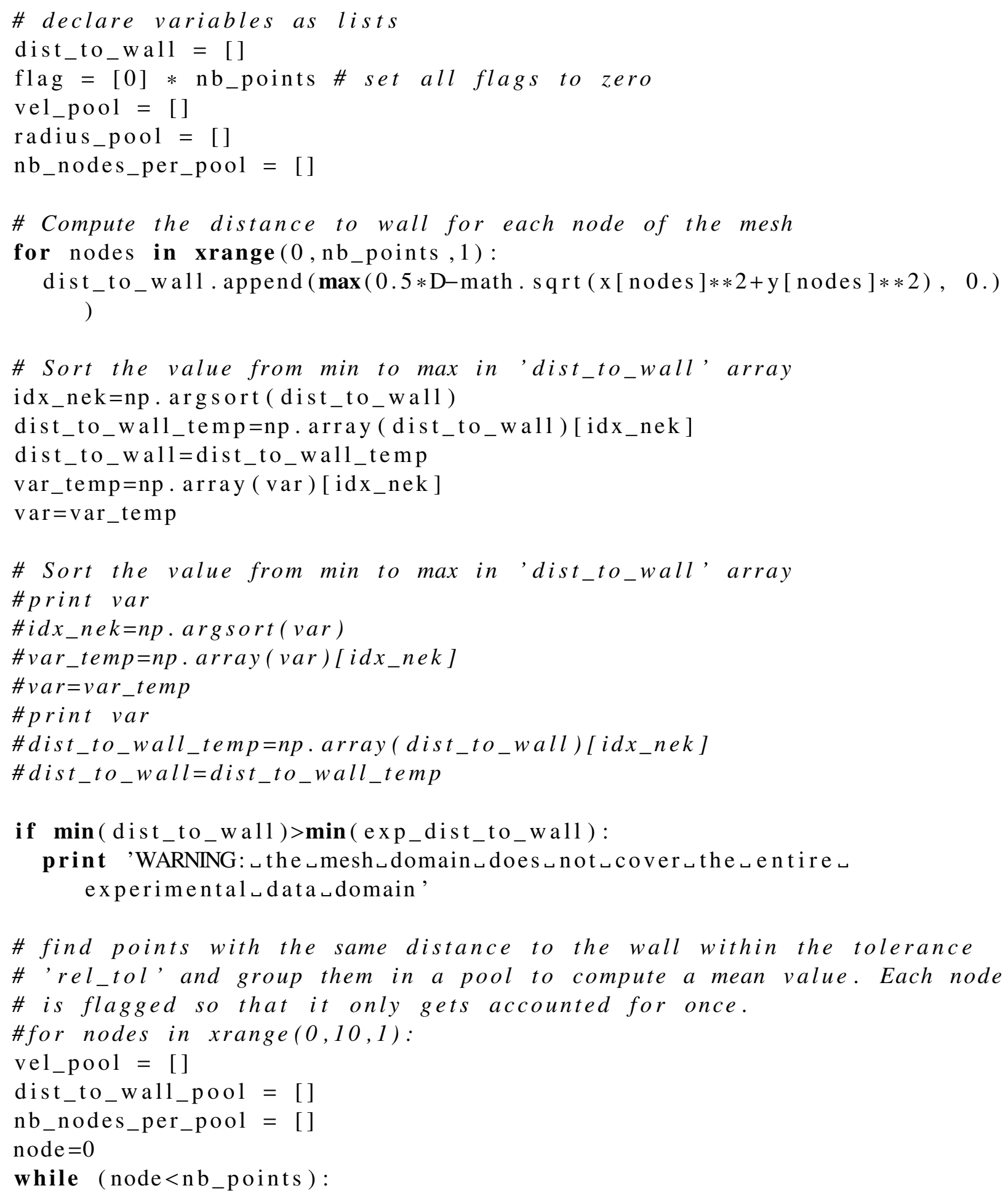




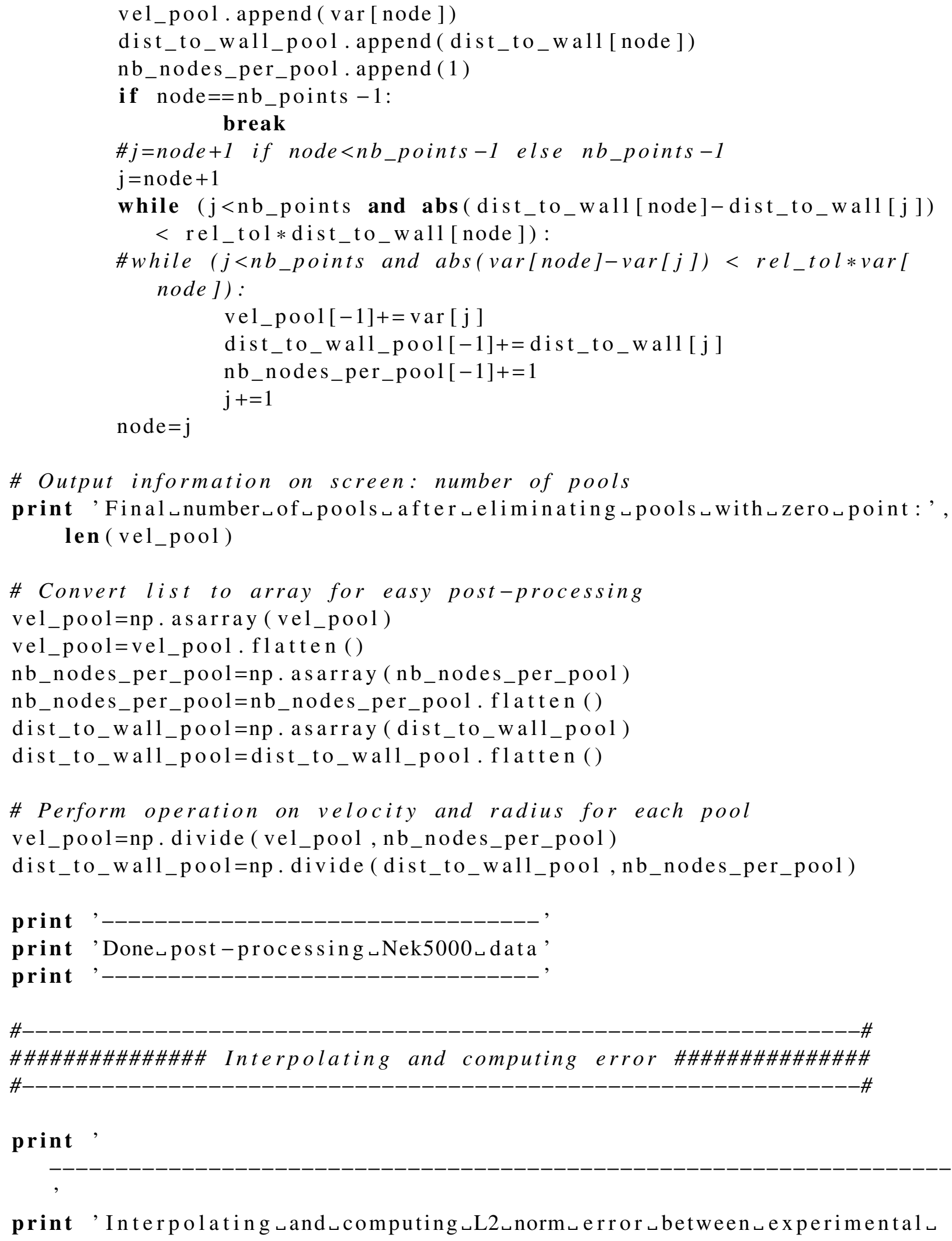


and $\left\llcorner\mathrm{Nek5000 \_ data}\right.$

print

\# Get Nek5000 data at exoerimental nodes by interpolating

print 'Interpolating data_at', len(exp_dist_to_wall), 'points'

nek5000_vel_pool_at_exp_nodes=np.interp (exp_dist_to_wall,

dist_to_wall_pool, vel_pool)

nek5000_vel_pool_at_exp_nodes=np.array (nek5000_vel_pool_at_exp_nodes)

\# Compute L2 error norm

sri=math.sqrt (np.1inalg.norm (exp_vel-nek5000_vel_pool_at_exp_nodes )

print 'Compute $\mathrm{L}_{\llcorner}$error norm: $^{\prime}$, sri

\section{\# Saving plot}

plt.plot (exp_dist_to_wall, exp_vel, '*', label='experimental_data')

plt.plot (dist_to_wall_pool, vel_pool, 'x', label='Nek5000॰poolıdata')

plt.plot (exp_dist_to_wall, nek5000_vel_pool_at_exp_nodes, '+', label='

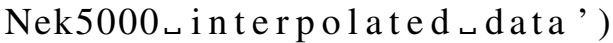

legend $=$ plt.legend $($ loc $=$ 'lower $\sqcup$ right', shadow $=$ True $)$

frame $=$ legend.get_frame ()

frame.set_facecolor ('0.90')

for label in legend.get_texts ():

label.set_fontsize('large')

for label in legend.get_lines ():

label.set_linewidth (1.5)

plt. suptitle ('Res31000', fontsize =20)

plt.xlabel ('Distance $\leftarrow$ to॰wall', fontsize $=18$ )

plt.ylabel('Velocity magnitude', fontsize =16)

fig_name $=$ 'Re-31k-'+num+'.eps'

plt. savefig (fig_name, format $=$ 'eps', dpi=1000)

print 'Saving plot॰using $_{\llcorner}$Matplotlib:' , fig_name

output_data_txt_file="velocity - data $-"+$ num+".txt"

with open( output_data_txt_file, "w") as fin :

header="\#\#_Nek5000-dist -to-wall "+" \t "+"Nek5000-velocity "+" \t"+"

intepolated-velocity"+" \t"+"exp-dist-to-wall"+"\t"+"exp-velocity \#\#"+"\n"

fin. write (header)

for e in it.izip_longest(dist_to_wall_pool, vel_pool, nek5000_vel_pool_at_exp_nodes, exp_dist_to_wall, exp_vel, fillvalue $=$, , ) :

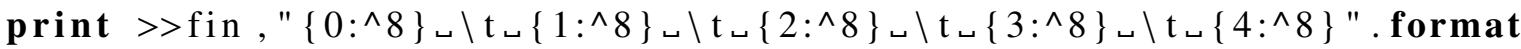
$(* \mathrm{e})$ 


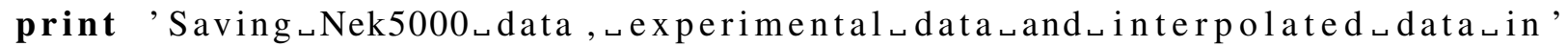
, output_data_txt_file

\section{print}

,

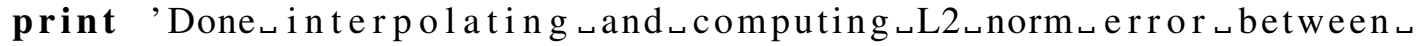
experimental $\llcorner$ and $\lrcorner \mathrm{Nek}_{5000}$ data print

,

with open(dakota_output_file,"a+") as f :

f. write (str ( sri))

data.close ()

exp_data.close ()

sys.exit () 